\section{Mechanisms of thrombocytopenia in platelet-type von Willebrand disease}

\author{
Loredana Bury, ${ }^{1}$ Alessandro Malara, ${ }^{2,3}$ Stefania Momi, ${ }^{1}$ Eleonora Petito, ${ }^{1}$ \\ Alessandra Balduini ${ }^{2,3}$ and Paolo Gresele ${ }^{1}$
}

${ }^{1}$ Department of Medicine, Section of Internal and Cardiovascular Medicine, University of Perugia; ${ }^{2}$ Department of Molecular Medicine, University of Pavia and ${ }^{3}$ Biotechnology Research Laboratories, IRCCS San Matteo Foundation, Pavia, Italy

\section{ABSTRACT}

$\mathrm{P}$ atelet-type von Willebrand disease is an inherited platelet disorder characterized by thrombocytopenia with large platelets caused by gain-of-function variants in GP1BA leading to enhanced GPIb $\alpha$-von Willebrand factor (vWF) interaction. GPIb $\alpha$ and vWF play a role in megakaryocytopoiesis, thus we aimed to investigate megakaryocyte differentiation and proplatelet-formation in platelet-type von Willebrand disease using megakaryocytes from a patient carrying the Met239Val variant and from mice carrying the Gly233Val variant. Platelet-type von Willebrand disease megakaryocytes bound vWF at an early differentiation stage and generated proplatelets with a decreased number of enlarged tips compared to control megakaryocytes. Moreover, they formed proplatelets upon contact with collagen, differently from normal megakaryocytes. Similarly, collagen triggered megakaryocytes showed defective activation of the RhoA-MLC2 axis, which prevents proplatelet formation, and increased phosphorylation of Lyn, which acts as a negative regulator of GPVI signaling, thus preventing ectopic proplatelet-formation on collagen. Consistently, human and murine bone marrow contained an increased number of extravascular platelets compared to controls. In addition, platelet survival of mutant mice was shortened compared to control mice, and the administration of desmopressin, raising circulating vWF, caused a marked drop in platelet count. Taken together, these results show for the first time that thrombocytopenia in platelet-type von Willebrand disease is due to the combination of different pathogenic mechanisms, i.e. the formation of a reduced number of platelets by megakaryocytes, the ectopic release of platelets in the bone marrow, and the increased clearance of platelet/vWF complexes.

\section{Introduction}

Platelet-type von Willebrand disease (PT-vWD) is an autosomal dominant inherited bleeding disorder caused by gain-of-function variants of GP1BA conferring enhanced affinity for von Willebrand factor (vWF) to platelet integrin GPIb $\alpha .{ }^{1}$ This disorder is characterized by a mild to moderate bleeding phenotype ${ }^{2}$ associated with fluctuating thrombocytopenia, which is conventionally explained by the formation of vWF-platelet complexes that are rapidly cleared from the circulation. ${ }^{1}$ Findings in support of this interpretation are the presence of circulating vWFplatelet complexes in a murine model of $\mathrm{PT}-\mathrm{vWD}^{3}$ and the increased platelet clearance of mice with type $2 \mathrm{~B}-\mathrm{vWD}$, a condition in which mutated vWF shows enhanced affinity for GPIb $\alpha .{ }^{4}$ No studies, however, have shown enhanced platelet clearance in either patients or mice with PT-vWD.

On the other hand, GPIb $\alpha$ is an important regulator of megakaryocytopoiesis, as shown by defective proplatelet formation (PPF) by megakaryocytes incubated with GPIb $\alpha$-blocking antibodies ${ }^{5}$ and by megakaryocytes from Bernard-Soulier syndrome patients. ${ }^{6,7}$ Indeed, GPIb $\alpha$ regulates the polarization of the megakaryocyte demarcation membrane system and transendothelial platelet biogenesis through intracellular signals that involve the small GTPases Cdc42/RhoA. ${ }^{8}$

von Willebrand factor also plays a role in megakaryocytopoiesis, by accelerating and boosting $\mathrm{PPF}^{9}$ and by increasing platelet production upon megakaryocyte expo-
Ferrata Storti Foundation

Haematologica 2019

Volume 104(7):1473-1481

\section{Correspondence:}

PAOLO GRESELE

paolo.gresele@unipg.it

Received: June 22, 2018.

Accepted: January 11, 2019.

Pre-published: January 17, 2019.

doi:10.3324/haematol.2018.200378

Check the online version for the most updated information on this article, online supplements, and information on authorship \& disclosures: www.haematologica.org/content/104/7/1473

(C)2019 Ferrata Storti Foundation

Material published in Haematologica is covered by copyright. All rights are reserved to the Ferrata Storti Foundation. Use of published material is allowed under the following terms and conditions:

https://creativecommons.org/licenses/by-nc/4.0/legalcode. Copies of published material are allowed for personal or internal use. Sharing published material for non-commercial purposes is subject to the following conditions:

https://creativecommons.org/licenses/by-nc/4.0/legalcode, sect. 3. Reproducing and sharing published material for commercial purposes is not allowed without permission in writing from the publisher. 
sure to high shear rates. ${ }^{10,11}$ Megakaryocytes from type 2B$\mathrm{vWD}$ patients form a reduced number of abnormally large proplatelets, ${ }^{9,12}$ explaining the macrothrombocytopenia associated with this condition and suggesting that an enhanced GPIb $\alpha$-vWF interaction may alter PPF. It is, therefore, conceivable that defective PPF may contribute to thrombocytopenia in PT-vWD.

Besides GPIb $\alpha$, other megakaryocyte receptors for adhesive proteins play an important role in the regulation of PPF. ${ }^{13}$ In particular, the interaction of $\alpha_{2} \beta_{1}$ and GPVI with type I collagen inhibits PPF, in this way preventing ectopic platelet release in the bone marrow endosteal niche. The interaction of megakaryocyte $\alpha_{2} \beta_{1}$ with type I collagen activates the Rho-ROCK pathway which induces the phosphorylation of myosin light chain 2 (MLC2), ${ }^{14}$ thus inhibiting PPF, while GPVI triggers inhibitory signaling mediated by Src family kinases (SFK), ${ }^{15}$ a family of kinases acting on an array of downstream effectors, including adaptor, enzyme, and cytoskeletal proteins, that collectively co-ordinate cytoskeletal remodeling. ${ }^{16}$

The loss of physiological suppression of PPF by type I collagen, and consequently the ectopic release of platelets in the bone marrow, have been reported to cause thrombocytopenia in $\mathrm{WAS}^{-/}$mice (a model of Wiskott-Aldrich syndrome $)^{17}$ and in patients with $M Y H 9-\mathrm{RD}^{18}{ }^{18}$ two inherited disorders characterized by reduced platelet number.

We studied proplatelet formation using human and murine PT-vWD megakaryocytes and show that vWF is bound to megakaryocyte surface GPIb $\alpha$ at early differentiation stages. We also show that megakaryocytes form a reduced number of large platelets compared to control megakaryocytes. Moreover, suppression of proplatelet formation by type I collagen is impaired and associates with abnormalities of the intracellular signaling pathways triggered by collagen, involved in the suppression of proplatelet formation. An enhanced number of free platelets were consistently observed in the bone marrow of PTvWD mice. Increased clearance of platelet/vWF complexes is also evident, and it contributes to reduce the platelet count, especially in stress conditions in which circulating vWF levels are increased.

\section{Methods}

Throughout the manuscript the terms control and PT-vWD will refer to human megakaryocytes/platelets and the terms $\mathrm{Tg}^{\mathrm{WT}}$ and $\mathrm{Tg}^{\mathrm{G} 233 \mathrm{~V}}$ to murine megakaryocytes/platelets.

This study was approved by the ethics committee Comitato Etico Aziende Sanitarie (CEAS) of the Region of Umbria (approval number 2663/15).

\section{Animals}

The generation of mice expressing the human GPIb $\alpha$ transgene carrying the G233V variant in homozygous form $\left(\mathrm{Tg}^{\mathrm{G} 233 \mathrm{~V}}\right)$ and of control mice expressing a wild-type human GPIb $\alpha$ transgene $\left(\mathrm{Tg}^{\mathrm{WT}}\right)$ has been previously described. ${ }^{3,19}$ These animals express a human GPIb $\alpha$ transgene and no mouse GPIb $\alpha$, and both $\mathrm{Tg}^{\mathrm{G} 233 \mathrm{~V}}$ and $\operatorname{Tg}^{\mathrm{WT}}$ have been consistently backcrossed with C57BL/6J animals.

\section{Human and murine megakaryocyte cultures}

To obtain human megakaryocytes, $\mathrm{CD} 45^{+}$or alternatively $\mathrm{CD} 34^{+}$cells were separated from peripheral blood of a PT-vWD patient carrying the Met239Val mutation ${ }^{20}$ (studied on 12 different occasions) and of 15 healthy controls. The obtained cells were then induced to differentiate into megakaryocytes in StemSpan serum free expansion medium (SFEM) supplemented with human recombinant stem cell factor $(\mathrm{SCF})(25 \mathrm{ng} / \mathrm{mL})$ and thrombopoietin (TPO) $(10 \mathrm{ng} / \mathrm{mL})$ for seven days and TPO alone for the following seven days, as previously described. ${ }^{5-7,21,22}$ All subjects gave their informed consent and all studies were carried out according to the principles of the Declaration of Helsinki. Murine megakaryocytes were cultured from bone marrow cells flushed from mouse femurs in Dulbecco's modified eagle's medium (DMEM) supplemented with $10 \%$ fetal calf serum (FCS) and recombinant murine TPO (10 ng/mL) for four days, as previously described. ${ }^{22}$ For details see the Online Supplementary Appendix.

\section{Megakaryocyte spreading and proplatelet formation}

Spreading and proplatelet formation in human and murine megakaryocytes were evaluated by immunofluorescence, as previously described. ${ }^{5-7,21,22}$ For details see the Online Supplementary Appendix.

\section{von Willebrand factor binding to megakaryocytes}

Exogenous vWF was not added to the medium in any of the experiments. vWF binding to human and murine megakaryocytes was assessed by confocal microscopy ${ }^{5}$ and by flow cytometry. ${ }^{20,23}$ For details see the Online Supplementary Appendix. vWF secretion by megakaryocytes was measured in cell culture supernatants at days 3, 7 and 14 of cell differentiation using an ELISA kit (Asserachrom VWF:Ag, Stago Italia, Milan, Italy).

\section{Megakaryocyte intracellular signaling triggered by type I collagen}

Megakaryocytes were plated for 16 hours (h) in 12-well plates pre-coated with $25 \mu \mathrm{g} / \mathrm{mL}$ of type I collagen or $1 \%$ BSA and then lysed in HEPES-glycerol lysis buffer (HEPES $50 \mathrm{mM}, 10 \%$ glycerol, 1\% Triton X-100, MgCl21.5 mM, EGTA $1 \mathrm{mM}, 1 \%$ protease inhibitors). RhoA activity (RhoA-GTP), the phosphorylation of MLC2, of Src-family kinases (SFK) and of Lyn were assessed by Western blotting. ${ }^{24}$ For details see the Online Supplementary Appendix.

\section{Megakaryocyte migration assay}

Megakaryocyte migration assay was performed as described ${ }^{25}$ in transwell migration chambers (8 $\mu \mathrm{m}$, Millipore) coated or not with $25 \mu \mathrm{g} / \mathrm{mL}$ type I collagen, and cells were counted by flow cytometry. ${ }^{26}$ Results were expressed as chemotaxis index (CI). ${ }^{27,28}$ For details see the Online Supplementary Appendix.

\section{Bone marrow histology}

Immunostaining of platelets was carried out in ten sections of human bone marrow from the PT-vWD patient, from three patients with immune thrombocytopenia (ITP), and from three controls and ten sections of murine bone marrow from femurs and tibiae of $3 \mathrm{Tg}^{\mathrm{WT}}$ and $3 \mathrm{Tg}^{\mathrm{G} 233 \mathrm{~V}}$ mice. ${ }^{27,28}$ For details see the Online Supplementary Appendix.

\section{Measurement of platelet life span in mice}

Mice were injected intravenously with $0.5 \mu \mathrm{g} / \mathrm{g}$ body weight of an anti-GPIX mAb (Emfret Analytics, Eibelstadt, Germany) conjugated with DyLight 488 (Life Technologies, Italia) and the percentage of residual fluorescent platelets was assessed for five days by flow cytometry as described. ${ }^{4,26}$ Blood was obtained from tail tip to cuts in tubes containing $4 \%$ sodium citrate.

The same experiments were repeated by administering to mice desmopressin (DDAVP) $(0.3 \mu \mathrm{g} / \mathrm{kg})$ by subcutaneous injection immediately after intravenous injection of the DyLight 488-conju- 
gated anti-GPIX mAb. For details see the Online Supplementary Appendix.

\section{von Willebrand factor-bound circulating platelets}

Blood smears were prepared from five different samples of EDTA-anticoagulated human or mouse blood from cardiac puncture and analyzed by fluorescence microscopy, as previously described. ${ }^{21,22}$ For details see the Online Supplementary Appendix.

\section{Statistical analysis}

Data are presented as means \pm Standard Error of Mean (SEM). The $t$-test for unpaired data was used to analyze results. $P<0.05$ was considered statistically significant.

\section{Results}

Platelet-type von Willebrand disease patient

The characteristics of the PT-vWD patient carrying the Met239Val variant in this study have been reported previously. ${ }^{20}$ Platelet count was mildly and variably reduced (41-168 $\left.\times 10^{9} / \mathrm{L}\right)$ and platelet volume mildly increased (13.5 $\mathrm{fL}$, normal: 8.0-12.0 fL), with 94\% normal, 5\% large, and $1 \%$ giant platelets (normal: $95-100 \% ; 0-4 \%$ and $0-1 \%$, respectively). ${ }^{29}$ Similar to the platelet count, the percentage of reticulated platelets was highly heterogeneous, ranging from $5.7 \%$ to $23.6 \%$ (normal values $7-10 \%$ ). The lowest platelet counts $\left(41\right.$ and $\left.80 \times 10^{9} / \mathrm{L}\right)$ and the highest

A
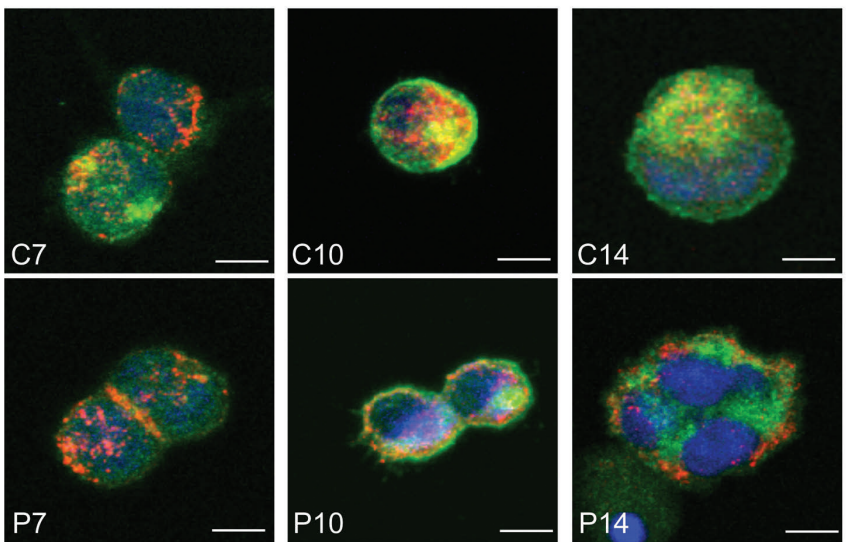

B

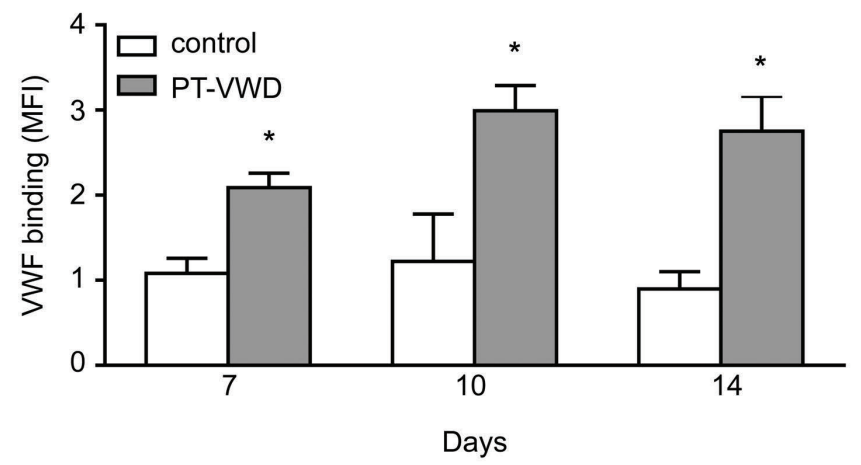

C

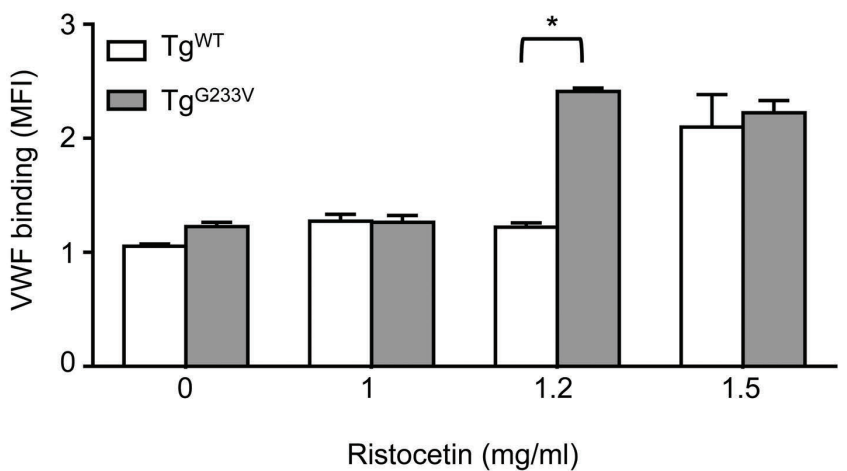

Figure 1. von Willebrand factor (VWF) binding to megakaryocytes. (A) vWF binding to human megakaryocytes at different days of culture as assessed by confocal microscopy. vWF binding was analyzed by confocal microscopy of megakaryocytes cytospun on glass coverslips. vWF is stained green (Alexa Fluor 488 Goat AntiRabbit IgG), CD42b is stained red (Alexa Fluor 568 donkey Anti-mouse IgG), and nuclei are stained blue with Hoechst. Samples were mounted using the ProLong Antifade medium (Molecular Probes) and analyzed at room temperature using a TCS SPII confocal laser system equipped with a DM IRBE inverted microscope and a 40x OIL NA objective (Leica, Bensheim, Germany). Representative merging images are shown. Surface localization of vWF was established by analyzing the intensity of the fluorescence signal along the $x$-axis on the optical section for each fluorochrome using Image J software. Scale bars=20 um. P: PT-vWD megakaryocytes; C: control megakaryocytes. Representative images are of megakaryocytes from three controls and of three different preparations from the PT-vWD patient. (B) Binding of vWF (mean fluorescence intensity, MFI) to human resting control and PTvWD megakaryocytes at days 7,10 and 14 of differentiation, as assessed by flow cytometry. Data represent mean+Standard Error of Mean (SEM) of five controls and five different preparations from the PT-VWD patient $(* P<0.05$ vs. control). Raw FACS data are shown in Online Supplementary Figure S11A. (C) Binding of vWF to murine megakaryocytes from $\mathrm{Tg}^{\mathrm{WT} T}$ and $\mathrm{Tg}^{\mathrm{G} 233 \mathrm{v}}$ mice at day 4 of differentiation induced by increasing doses of ristocetin, as assessed by flow cytometry. Data represent mean \pm SEM for five repeated measures $(* P<0.05$ vs. 0). Raw FACS data are shown in Online Supplementary Figure S11B. 
reticulated platelet percentages $(16.6 \%$ and $23.6 \%)$ corresponded to periods in which the patient was pregnant. Her bone marrow biopsy showed an increased number of megakaryocytes (Online Supplementary Figure S1).

\section{von Willebrand factor binding to megakaryocytes}

von Willebrand factor bound to the surface of human PT-vWD megakaryocytes, co-localizing with GPIb $\alpha$, was detected by confocal microscopy (Figure 1A) and flow cytometry (Figure 1B) on days 7, 10 and 14 of cell differentiation, while control megakaryocytes did not show bound vWF at any of the differentiation times. vWF did not co-localize with GPIba intracellularly (Online Supplementary Figure S2). vWF was secreted from megakaryocytes starting from day 7 until day 14 of differentiation (Online Supplementary Figure S3A).

von Willebrand factor was not detected on the surface of megakaryocytes from $\mathrm{Tg}^{\mathrm{G} 233 \mathrm{~V}}$ mice (Online Supplementary Figure S3B), in line with previous observations. ${ }^{19}$ It cannot be excluded that $\mathrm{vWF}$ is bound to murine mutant megakaryocytes in vivo but that the quantity is too low to be detected by immunofluorescence, or that vWF bound to the megakaryocyte surface is lost during murine megakaryocyte isolation, culture and analysis. However, megakaryocytes from $\operatorname{Tg}^{\mathrm{G} 233 \mathrm{~V}}$ mice bound vWF after stimulation with a lower dose of ristocetin compared to megakaryocytes from control mice, confirming increased affinity for vWF (Figure 1C).

\section{Megakaryocyte differentiation, spreading, proplatelet formation and migration}

The percentage of peripheral-blood derived $\mathrm{CD} 45^{+} / \mathrm{CD} 34^{+}$cells differentiating in megakaryocytes was comparable in PT-vWD and controls (PT-vWD $37.3 \pm 7.2 \%$, controls $41.2 \pm 9.3 \%$ ), with a slightly but significantly lower percentage of PT-vWD megakaryocytes reaching stage IV of differentiation (Figure 2A). Transmission electron microscopy of PT-vWD megakaryocytes did not show ultrastructural abnormalities (Online Supplementary Figure S4).

The fraction of megakaryocytes spreading on immobilized fibrinogen, vWF or type I collagen was similar in PTvWD and controls (Online Supplementary Figure S5A and B). The fraction of megakaryocytes generating proplatelets, either in suspension or on fibrinogen- or VWF-coated coverslips, was also comparable in PT-vWD and controls
A

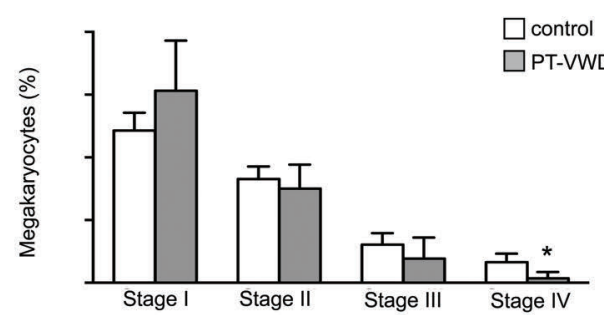

B

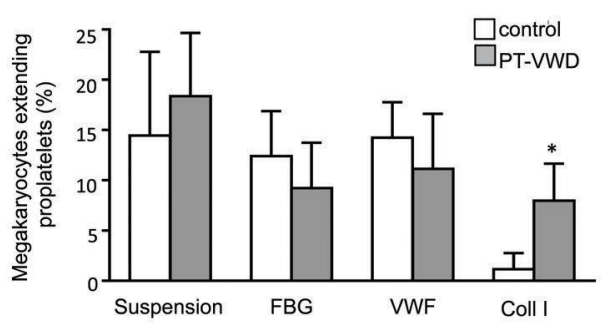

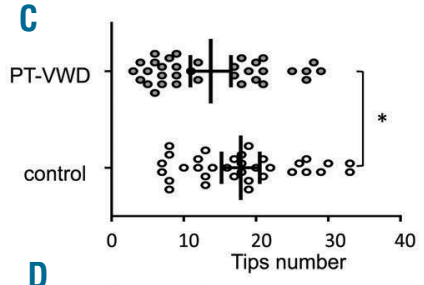

D

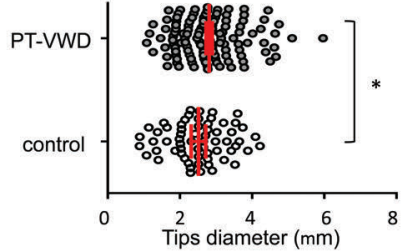

E
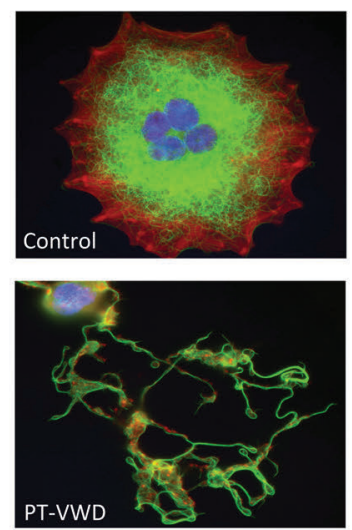

$\mathrm{F}$

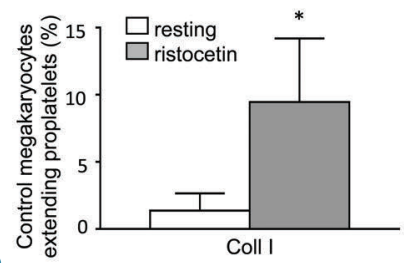

G

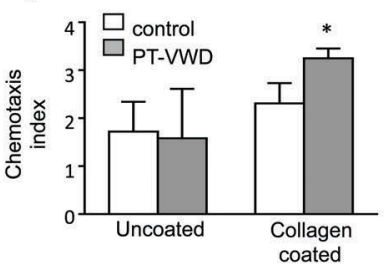

Figure 2. Proplatelet formation from human megakaryocytes. (A) Megakaryocyte percentage and maturation. The percentage of CD41 cells at day 14 of culture was measured by flow cytometry. Maturation of megakaryocytes was determined by fluorescence microscopy based on ploidy, cell diameter, and CD41 expression. For each sample, at least 100 megakaryocytes were evaluated. Data represent mean \pm Standard Deviation (SD) for five controls and for the platelet-von Willebrand disease (PT-vWD) patient on five different occasions; ${ }^{*} P<0.05$ vs. control. (B) Percentage of megakaryocytes-extending proplatelets in suspension, or onto glass coverslips coated with fibrinogen (FBG), vWF or type I collagen (Coll I). Data represent mean \pm Standard Error of Mean (SEM) of five controls and of five different preparations from the PT-vWD patient ( $* P<0.05$ vs. controls). (C) Representative images of control and PT-vWD megakaryocytes plated on type I collagen. Scale bars $=20$ $\mu \mathrm{m} . \beta 1$ tubulin is stained green (Alexa Fluor ${ }^{\circledR} 488$ Goat Anti-Rabbit IgG; Molecular Probes, Life Technologies, Milan, Italy), polymerized actin is stained red (rhodamine-phalloidine; Molecular Probes), and nuclei are stained blue with Hoechst. Specimens were mounted with the ProLong Antifade medium (Molecular Probes), analyzed at room temperature with a Carl Zeiss Axio Observer.A1 fluorescence microscope (Carl Zeiss Inc., Oberkochen, Germany) using a $63 x / 1.4$ Plan-Apochromat oil-immersion objective and images acquired using the AxioVision software (Carl Zeiss Inc.). All polynucleated cells extending protrusions with terminal tips were defined as proplatelet-forming megakaryocytes while those displaying a flattened shape with actin organized into focal adhesion points and fibers as spreading megakaryocytes. Scale bars $=20 \mu \mathrm{m}$. (D) Number of proplatelet tips generated by megakaryocytes. Individual data, means and $95 \% \mathrm{Confidence} \mathrm{Interval} \mathrm{(95 \% Cl)} \mathrm{are}$ shown ( $* P<0.05$ vs. control). Measures were carried out on megakaryocytes from five controls and five different preparations from the PT-vWD patient. (E) Diameter of proplatelet tips generated by megakaryocytes. Individual data, means and $95 \% \mathrm{Cl}$ are shown ( $P<0.05$ vs. control). Measures were carried out on megakaryocytes from five controls and five different preparations from the PT-vWD patient. (F) Percentage of control megakaryocytes-extending proplatelets on type I collagen (Coll I) under resting conditions or after incubation with $1.5 \mathrm{mg} / \mathrm{mL}$ of ristocetin. Data represent mean $\pm \mathrm{SEM}$ of five different experiments (*P<0.05 vs. resting). (G) Migration of megakaryocytes through transwell filters uncoated or coated with type I collagen in response to SDF- $1 \alpha(100 \mathrm{ng} / \mathrm{mL})$. Chemotaxis index (Cl) expresses the number of cells that have passed through the filter in response to SDF- $1 \alpha$ divided by the number of cells passed in the absence of SDF-1 $\alpha$ ( $n=4$; $* P<0.05$ vs. control). Measures were carried out on megakaryocytes from four controls and four different preparations from the PT-vWD patient. 
(Figure 2B and Online Supplementary Figure S5A). PT-vWD megakaryocytes extended very long and branched proplatelets on fibrinogen and vWF, probably due to the boosting role of bound $\mathrm{vWF}$ on proplatelet formation. ${ }^{9-11}$ However, the number of proplatelet tips was reduced and tip diameter was larger in PT-vWD megakaryocytes. In particular, we identified a subset of cells producing a number of tips below the $95 \%$ Confidence Interval (95\% CI) of controls, and which had tips of a diameter above the 95\% CI of controls (Figure 2C and D). Moreover, a significantly higher number of PT-vWD megakaryocytes extended proplatelets when plated on type I collagen, compared to control megakaryocytes (Figure $2 \mathrm{~B}$ and $\mathrm{E}$ ). Comparable results were obtained using mouse $\mathrm{Tg}^{\mathrm{G} 233 \mathrm{~V}}$ megakaryocytes (Online Supplementary Figure S6).

In order to exclude the possibility that defects of the col-
A
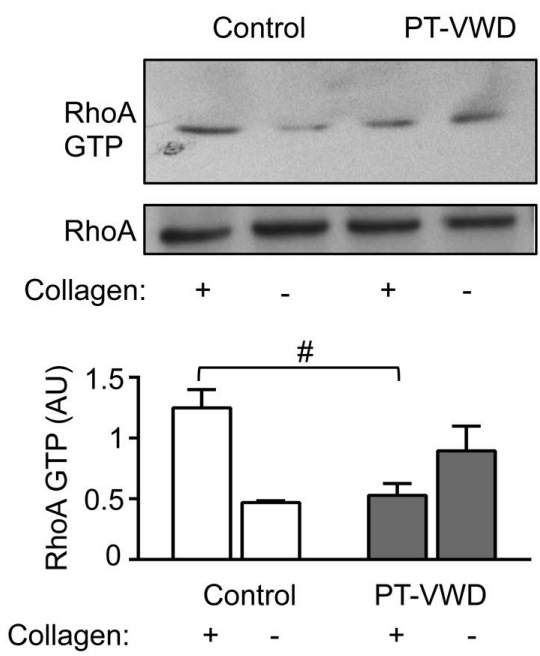

C
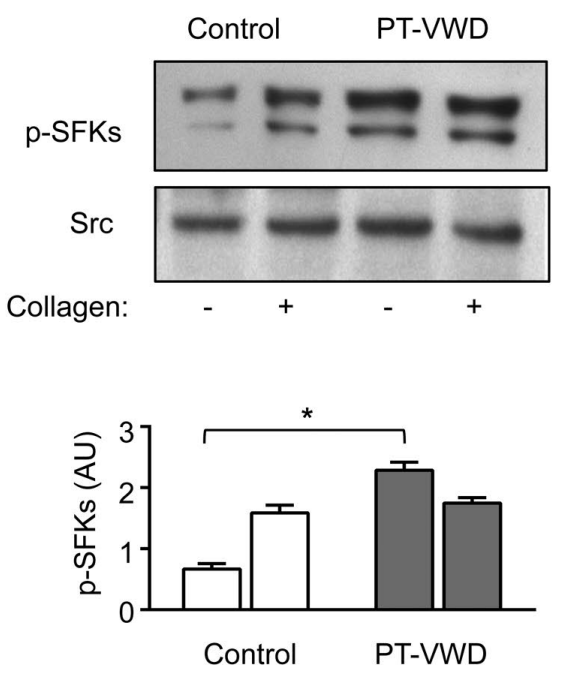

Collagen:

$-+$

$-\quad+$
B

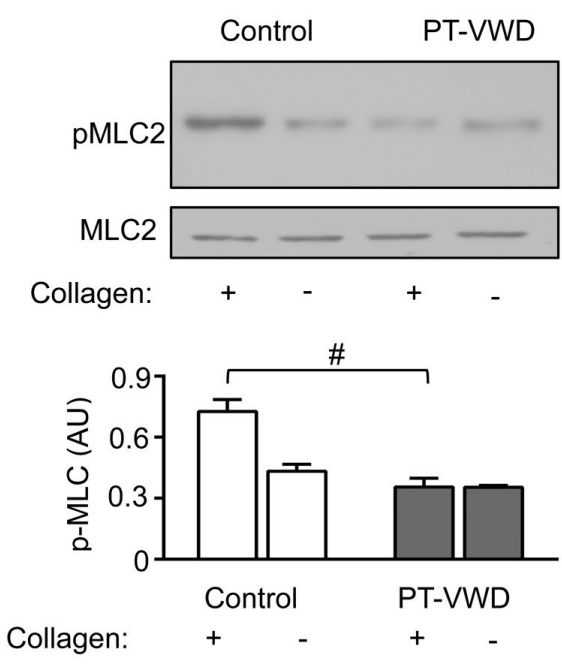

D
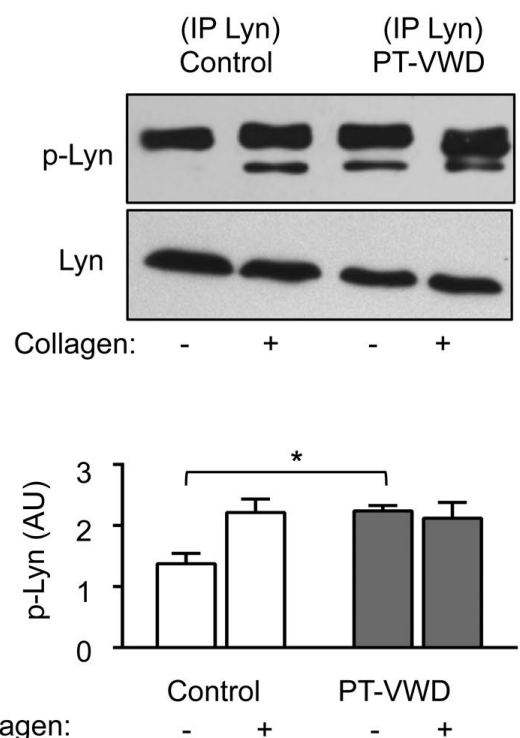

Figure 3. Collagen-triggered signaling in human megakaryocytes. (A) RhoA activation (RhoA-GTP) of megakaryocytes in suspension (-) or after 16 hours (h) of adhesion to type I collagen (+). Densitometric analysis was performed using the Image $\mathrm{J}$ software. Quantification of RhoA-GTP is relative to total RhoA expression and is expressed in arbitrary units (AU) ( $n=5$, \#Significantly different from control on type I collagen; $P<0.05)$. (B) MLC-2 phosphorylation (pMLC-2) of megakaryocytes in suspension (-) or after $16 \mathrm{~h}$ of adhesion to type I collagen (+). Densitometric analysis was performed using Image J software. Quantification of pMLC-2 is relative to total MLC-2 expression and is expressed in arbitrary units (AU). ( $n=5$; \#Significantly decreased vs. control on type I collagen; $P<0.05)$. (C) SFK phosphorylation (p-Src Tyr416) in megakaryocytes in suspension (-) or after $16 \mathrm{~h}$ of adhesion to type I collagen (+). Densitometric analysis was performed using Image J software. Quantification of p-SFK is relative to total SFK expression and is expressed in arbitrary units (AU) ( $n=5$, * Significantly increased vs. control in suspension; $P<0.05)$. (D) Lyn phosphorylation (p-Lyn) in megakaryocytes in suspension (-) or after $16 \mathrm{~h}$ of adhesion to type I collagen (+). Lyn was immunoprecipitated and western blotting was carried out using the anti-SFK antibody. Densitometric analysis was performed using Image J software. Quantification of P-Lyn is relative to total Lyn expression and is expressed in arbitrary units $(\mathrm{AU})(\mathrm{n}=5$; * Significantly different from resting control; $P<0.05)$. Blots are representative of megakaryocytes from five controls and five different preparations from the platelet-type von Willebrand disease patient. 
lagen receptors were responsible for this peculiar phenotype, we sequenced the GP6, ITGA2 and ITGB1 genes in our PT-vWD patient. However, we did not find any rare pathogenic variant or any common genetic variant associated with decreased response to collagen. In addition, we assessed the expression of $\alpha_{2} \beta_{1}$ by flow cytometry, which was also normal (Online Supplementary Table S1).

To assess whether the constitutive binding of vWF to GPIb $\alpha$ was directly responsible for the increased PPF on type I collagen, we incubated control human megakaryocytes with ristocetin $(1.5 \mathrm{mg} / \mathrm{mL})$ to induce vWF-GPIb $\alpha$ binding, and evaluated their PPF on type I collagen. Control megakaryocytes incubated with ristocetin showed increased PPF on type I collagen, similar to PTvWD megakaryocytes (Figure 2F), suggesting that the binding of vWF triggers proplatelet formation on collagen.

A significantly higher percentage of human PT-vWD megakaryocytes migrated through transwells coated with type I collagen in response to SDF1- $\alpha$. In contrast, migration through uncoated transwells, compared to control human megakaryocytes (Figure 2G) or through transwells coated with vWF was normal (data not shown), confirming an altered interaction of PT-vWD megakaryocytes with type I collagen and not a generalized abnormality of migration.

\section{Megakaryocyte intracellular signaling triggered by type I collagen}

RhoA activation (RhoA-GTP formation) triggered by adhesion to collagen was lower in PT-vWD compared to control megakaryocytes (Figure 3A). Consistently, collagen-induced MLC2 phosphorylation, which depends on RhoA activation, ${ }^{14}$ was also impaired in PT-vWD megakaryocytes (Figure 3B). On the contrary, SFK phosphorylation was significantly higher in resting PT-vWD than in control megakaryocytes but, differently from control megakaryocytes, it did not increase further after adhesion to collagen (Figure 3C). Lyn showed the same activation pattern as SFK (Figure 3D). Comparable results were obtained using mouse $\operatorname{Tg}^{\mathrm{WT}}$ and $\mathrm{Tg}^{\mathrm{G} 233 \mathrm{~V}}$ megakaryocytes
A

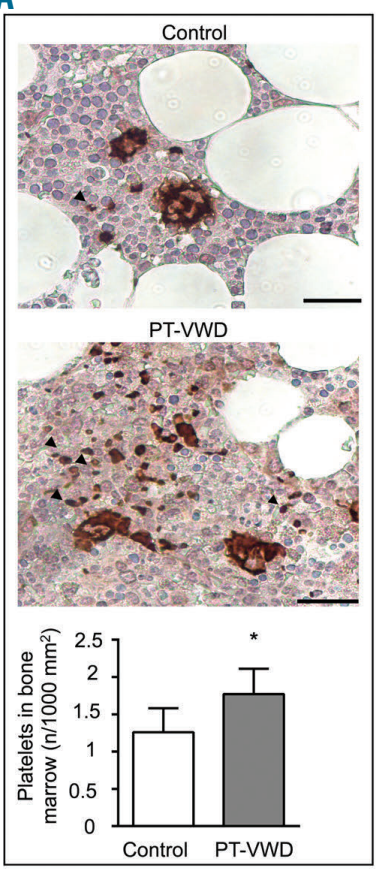

B

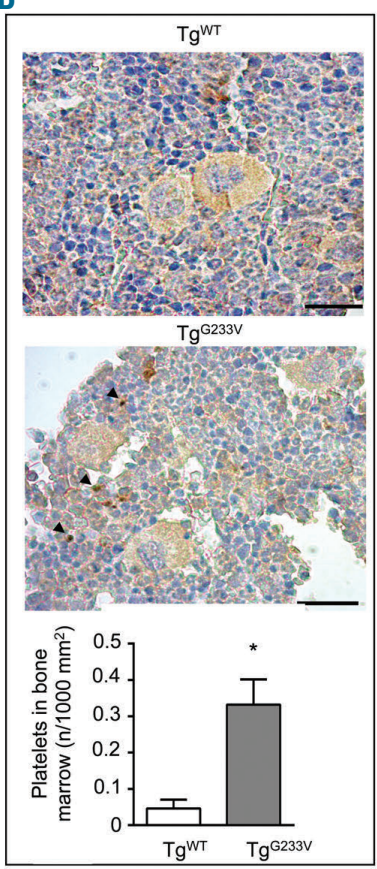

C

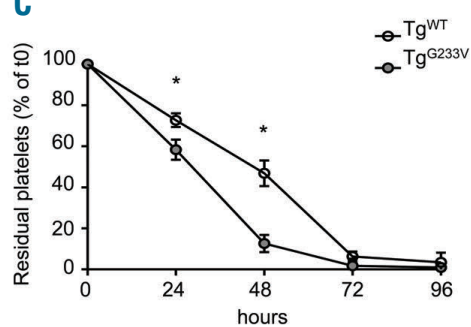

D
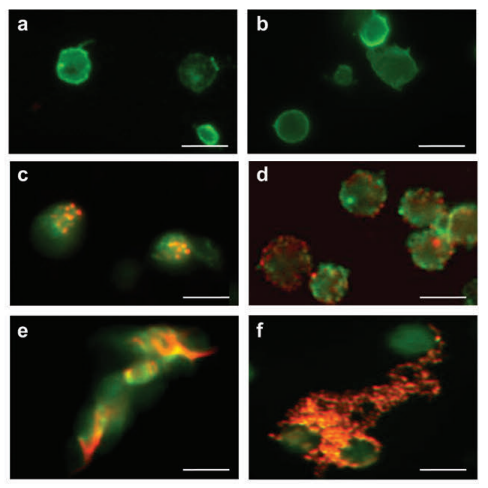

Figure 4. Platelets in bone marrow (BM) and platelet lifespan. (A) BM sections were stained using a primary rabbit-anti-human $\beta 1$-tubulin antibody and a secondary biotinylated goat anti-rabbit lgG thus highlighting megakaryocytes and platelets; specimens were counterstained with Hematoxylin \& Eosin (H\&E). Scale bars=40 $\mu \mathrm{m}$. Pictures were analyzed using Image J software and platelet number in BM was expressed as the number of platelets per 1000 um ${ }^{2}$ of cell-covered slide surface $\left({ }^{*} P<0.05\right.$ vs. control). Ten different human BM sections were analyzed from the platelet-type von Willebrand disease (PT-vWD) patient, and ten each from three immune thrombocytopenia (ITP) patients and from three healthy controls. (B) Murine BM biopsies of $\operatorname{Tg}^{\mathrm{WT}}$ and $\operatorname{Tg}^{\mathrm{G} 233 \mathrm{~V}}$ mice were stained using a rabbit-anti-mouse CD41 antibody and a secondary biotinylated goat anti-rabbit lgG; specimens were counterstained with hematoxylin \& eosin. Scale bars $=40$ um. Pictures were analyzed using Image J software and platelet number was expressed as the number of platelets per $1000 \mu \mathrm{m}^{2}$ of cells (right) (*P<0.05 vs. TgwT). Ten different BM sections were analyzed from each of three $\mathrm{Tg}^{\mathrm{WT}}$ and three $\mathrm{Tg}^{\mathrm{G} 233 \mathrm{~V}}$ mice. (C) $\mathrm{Tg}^{\mathrm{WT}}$ (white circles) and $\mathrm{Tg}^{\mathrm{G} 233 \mathrm{~V}}$ mice (gray circles) were injected with a DyLight 488 -conjugated antiGPIX mAb which confers to platelets a green fluorescence, and residual green fluorescent platelets were quantified by flow cytometry at different intervals from DyLight 488-conjugated anti-GPIX mAb injection. Data are expressed as the percentage of GPIX-positive platelets relative to the total CD41/61-PE positive platelet population with time $=0$ being arbitrarily set at $100 \%$. Data represent mean \pm Standard Error of Mean $(S E M)\left(n=4 ; * P<0.05\right.$ vs. Tg $\left.{ }^{W T}\right)$. (D) von Willebrand factor (vWF) binding to human (a, c, e) and murine (b, d, f) platelets from EDTA-anticoagulated blood smears as assessed by fluorescence microscopy. vWF was not detected on the platelet surface of healthy controls (a) and TgWT mice (b), while it was bound to the platelet surface of the PT-vWD patient (60-80\% of platelets) in the Tg(233v mouse (70-90\% of platelets) (c) and Tg ${ }^{6233 v}$ mice (d). Platelet clumps linked by vWF were present in the circulation of the platelet-vWD (PT-vWD) patient (e) and Tg $2333 \mathrm{v}$ mice (f) but not of healthy controls and Tg ${ }^{\mathrm{WT}}$ mice. vWF is stained red (Alexa Fluor ${ }^{\circledR} 568$ Goat Anti-Rabbit lgG), CD42b is stained green (Alexa Fluor ${ }^{\circledR} 488$ Goat Anti-rat IgG). Samples were mounted using the ProLong Antifade medium (Molecular Probes) and analyzed at room temperature using a Carl Zeiss Axio Observer.A1 fluorescence microscope (Carl Zeiss Inc., Oberkochen, Germany). Representative merging images are presented. Scale bars=5 $\mu \mathrm{m}$, C: healthy control, P: PT-vWD. Data refer to blood smears from five healthy controls, five different blood samples from the PT-vWD patient and from five $\operatorname{Tg}^{\mathrm{WT}}$ and five Tg ${ }^{\mathrm{G} 233 \mathrm{v}}$ mice. 
(Online Supplementary Figure S7). Interestingly, control megakaryocytes cultured in suspension showed phosphorylation of Lyn when incubated with ristocetin, thus confirming that the binding of vWF to GPIb $\alpha$ triggers Lyn phosphorylation (Online Supplementary Figure S8A). Finally, Cofilin, a protein that is phosphorylated by activated RhoA, ${ }^{30}$ was less phosphorylated upon adhesion to collagen in PT-vWD than in control megakaryocytes (Online Supplementary Figure S8B).

\section{Platelets in bone marrow}

Immunohistochemistry showed an increased number of platelets in the bone marrow of our PT-vWD patient compared to healthy controls (Figure 4A). In order to exclude the possibility that the increased number of platelets was simply due to the enhanced number of megakaryocytes in bone marrow (Online Supplementary Figure S1), we counted platelets in bone marrow from patients with ITP who also show increased megakaryocytes in bone marrow, but here the number of platelets was comparable to controls (Online Supplementary Figure S9A). Similarly, bone marrow from $\operatorname{Tg}^{\mathrm{G} 233 \mathrm{~V}}$ mice showed an increased number of platelets compared to bone marrow from $\mathrm{Tg}^{\mathrm{WT}}$ mice (Figure 4B). The ratio between bone marrow platelets and circulating platelets, as assessed by flow cytometry, was also significantly increased in $\operatorname{Tg}^{\mathrm{G} 233 \mathrm{~V}}$ mice (Online Supplementary Figure S9B).

\section{Platelet life span and platelet-bound vWF}

The number of circulating platelets was $572.3 \pm 32.6 \times 10^{9} / \mathrm{L}$ in $\mathrm{Tg}^{\mathrm{WT}}$ mice and $180.1 \pm 47.2 \times 10^{9} / \mathrm{L}$ in $\mathrm{Tg}^{\mathrm{G} 233 \mathrm{~V}}$ mice. $\mathrm{Tg}^{\mathrm{G} 233 \mathrm{~V}}$ mice showed a reduced platelet half- life (26 hours) compared to $\mathrm{Tg}^{\mathrm{WT}}$ mice $(47 \mathrm{~h})$. A significantly lower percentage of DyLight 488-stained platelets was observed already $24 \mathrm{~h}$ after fluorescent anti-GPIX mAb injection, and the difference became even more evident 48 $\mathrm{h}$ after, when residual DyLight 488-stained platelets in blood were $12 \%$ in $\mathrm{Tg}^{\mathrm{G} 233 \mathrm{~V}}$ and $46 \%$ in $\mathrm{Tg}^{\mathrm{WT}}$ mice (Figure 4C). Moreover, $71.3 \pm 12.2 \%$ and $83.2 \pm 14.6 \%$ of circulating human and murine PT-vWD platelets showed surfacebound $\mathrm{vWF}$, respectively, while no vWF could be detected on the platelet surface in controls. vWF bridged-platelet aggregates were also seen in PT-vWD, and not observed in controls (Figure 4D).

These data suggest that vWF/platelet complexes circulating in blood in PT-vWD patients are rapidly cleared from the circulation, thus reducing platelet lifespan. Indeed, the administration of DDAVP increased plasma vWF both in $\operatorname{Tg}^{\mathrm{WT}}$ and $\mathrm{Tg}^{\mathrm{G} 233 \mathrm{~V}}$ mice $(+97 \pm 21 \%$ and $+93 \pm 12 \%$, respectively), but it caused a drastic reduction of platelet count only in $\mathrm{TgG}^{233 \mathrm{~V}}$ mice (Figure $5 \mathrm{~A}$ ). The percentage of circulating DyLight 488-stained platelets was reduced by $30 \%$ in $\mathrm{Tg}^{\mathrm{G} 233 \mathrm{~V}}$ mice $60 \mathrm{~min}$ after DDAVP injection while it did not vary significantly in $\mathrm{Tg}^{\mathrm{WT}}$ mice, confirming that vWF-bound platelets in PT-vWD are rapidly cleared from the circulation (Figure 5B). PT-vWD platelets did not show increased exposure of phosphatidylserine under resting conditions $\%$ of Annexin V-positive platelets: PT-VWD, $4.1 \pm 0.6 \%$, controls, $4.6 \pm 2.7 \%$ ). From the comparative quantitative evaluation of our studies, it emerges that ectopic proplatelet formation is the mechanism that contributes the most to thrombocytopenia in PT-vWD (Online Supplementary Table S2).
A

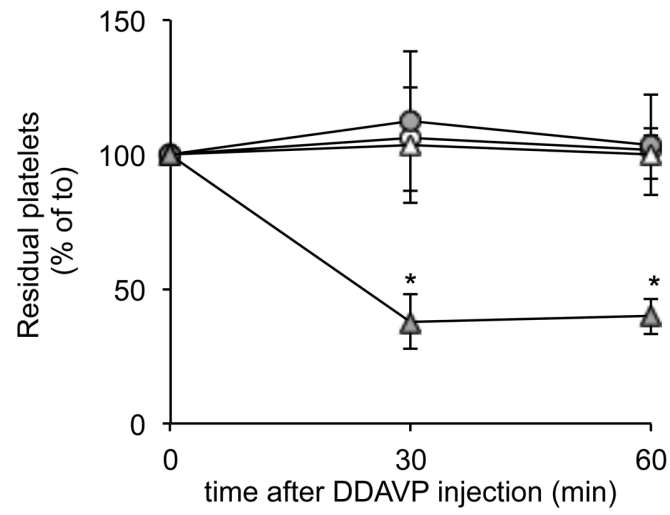

B

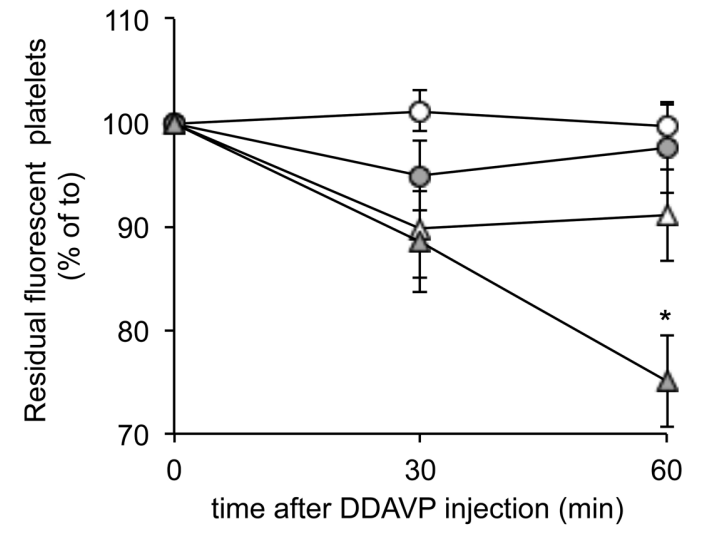

- $\mathrm{Tg}^{\mathrm{WT}}$ untreated

- $\operatorname{Tg}^{\mathrm{G} 233 \mathrm{~V}}$ untreated

$\rightarrow \mathrm{Tg}^{\mathrm{WT}}+$ DDAVP

$\rightarrow \mathrm{Tg}^{\mathrm{G} 233 \mathrm{~V}}+\mathrm{DDAVP}$

TgWT untreated

- Tg $^{\mathrm{G} 233 \mathrm{~V}}$ untreated

$\rightarrow \mathrm{Tg}{ }^{W T}+$ DDAVP

$\triangle \mathrm{Tg}^{\mathrm{G} 233 \mathrm{~V}}+\mathrm{DDAVP}$
Figure 5. Effect of desmopressin (DDAVP) administration on platelet count and lifespan in mice. (A) Platelet count was assessed by flow cytometry in saline-injected (circles) or DDAVP-injected (triangles) mice. A significant drop in platelet count was evident in Tg $^{\mathrm{G} 233 \mathrm{~V}}$ mice 30 minutes after the injection of DDAVP. Circulating platelets are expressed as percentage of the platelets measured at time $=0$. Data represent mean \pm Standard Error of Mean (SEM) $\left(n=5, * p<0.05\right.$ vs. Tg $\left.{ }^{\mathrm{wT}}\right)$. (B) Mice were infused with a DyLight 488-conjugated antiGPIX mAb. Residual green fluorescent platelets were quantified by flow cytometry in saline-injected (circles) or DDAVP-injected (triangles) mice. A significant drop in the percentage of fluorescent platelets was evident in $\operatorname{~g}^{\mathrm{G233} v}$ mice, but not in $\mathrm{Tg}^{\mathrm{WT}}$, 60 minutes after the injection of DDAVP. Circulating platelets are expressed as percentage of GPIX-positive platelets relative to the total CD41/61-PE positive platelet population measured at time $=0$. Data represent mean \pm SEM $(n=5$; $\star P<0.05$ vs. Tg $\left.^{\mathrm{WT}}\right)$. 


\section{Discussion}

The formation of proplatelets by megakaryocytes is tightly regulated by the interaction with bone marrow matrix proteins. In fact, several molecules, such as type IVcollagen, fibrinogen, fibronectin or vWF, support platelet biogenesis while type I-collagen inhibits it, thus preventing the premature release of platelets in the bone marrow. ${ }^{13}$ Defective PPF as a cause of inherited thrombocytopenias has been reported in Bernard-Soulier syndrome ${ }^{6,7}$ and in type 2B-vWD, ${ }^{9,12}$ but so far no studies have assessed PPF in PT-vWD.

Here we show that vWF binds to PT-vWD megakaryocytes at early stages of differentiation, causing a dysregulation of downstream signaling. This disturbs the suppression of PPF by type-I collagen, leading to the ectopic release of platelets in the bone marrow. Moreover, we show that megakaryocytes form a reduced number of large platelets, and that circulating PT-vWD platelets have vWF bound to their surface. We also observed that this leads to their clearance, especially when circulating levels of vWF increase. These mechanisms contribute to generate the thrombocytopenia typical of this disorder.

Differently from control megakaryocytes, which bind vWF only during PPF, PT-vWD megakaryocytes bind vWF at very early stages of differentiation. We show that, in vitro, differentiating megakaryocytes synthesize vWF and secrete it in the supernatant, with subsequent binding to mutant GPIba in PT-vWD. It is conceivable that this happens in vivo in the bone marrow, too.

Platelet-type von Willebrand disease megakaryocytes developed proplatelets with a reduced number of tips and slightly enlarged with respect to controls, in line with the mildly increased diameter of circulating platelets seen in patients. ${ }^{31}$ PPF requires a finely regulated cytoskeletal remodeling, ${ }^{13,22}$ and, given that GPIb $\alpha$ interacts with several cytoskeletal proteins, it is likely that mutated GPIb $\alpha$ leads to cytoskeletal perturbation with the formation of enlarged proplatelets.

Platelet-type von Willebrand disease megakaryocytes developed proplatelets on type I collagen, differently from control megakaryocytes. This abnormal behavior was reproduced with control megakaryocytes when vWF-GPIb $\alpha$ binding was induced by ristocetin.

Platelet-type von Willebrand disease megakaryocytes also migrated through type I collagen-coated transwells more than controls, further showing a deranged interaction with collagen. A recent study showed normal response to type I collagen of platelets form $\mathrm{Tg}^{\mathrm{C} 233 \mathrm{~V}}$ mice. ${ }^{32} \mathrm{However}$, in our experimental conditions, collagen was used as a substrate for adhesion of megakaryocytes and not in suspension as inducer of platelet aggregation, and this may account for the difference.

We did not detect defects of the collagen receptors, rare or common genetic variants of GP6, ITGA2 and ITGB1 by flow cytometry or by sequencing. Therefore, we investigated the signaling triggered by the interaction of megakaryocyte $\alpha_{2} \beta_{1}$ with collagen. ${ }^{14} \mathrm{We}$ found decreased RhoA activation (RhoA-GTP) and MLC2 phosphorylation, showing impaired $\alpha_{2} \beta_{1}$-mediated signaling. Interestingly, a dysregulation of the RhoA pathway has been recently shown in type $2 \mathrm{~B}-\mathrm{vWD}$, a condition associated with enhanced vWFGPIb $\alpha$ interaction. ${ }^{30} \mathrm{We}$ also assessed SFK phosphorylation, given that GPVI-dependent SFK signaling inhibits $\mathrm{PPF},{ }^{15}$ and found it to be increased in resting PT-vWD megakary- ocytes. Lyn, a SFK, exerts a dual function in platelets acting both as a positive and negative regulator of GPVI- and integrin-mediated activation. vWF binding to GPIb/V/IX triggers the association between the cytoplasmic tail of GPIb $\alpha$ and Lyn with consequent phosphorylation of the latter ${ }^{16,33}$ and downregulation of integrin and GPVI signaling. ${ }^{34,35}$ Indeed, Lyn phosphorylation (p-Lyn) was evident in resting PT-vWD megakaryocytes and was also triggered by ristocetin in control megakaryocytes. Thus, constitutive binding of vWF to GPIb $\alpha$ in PT-vWD megakaryocytes causes Lyn phosphorylation, and p-Lyn in turn acts as a negative regulator of GPVI- and $\alpha_{2} \beta_{1}$-triggered signaling, ${ }^{3437}$ thus preventing the inhibition of PPF by collagen (Online Supplementary Figure S10) with consequent ectopic PPF in the bone marrow causing thrombocytopenia. In accordance with this, we found an increased number of platelets in human and mouse PT-vWD bone marrow.

We also provide the first direct evidence of the presence of circulating platelet/vWF complexes in PT-vWD, associated in mice with a reduced platelet life span that exacerbates when the concentration of VWF in plasma is increased by DDAVP administration. These data confirm that the binding of vWF to the platelet surface accelerates platelet clearance. Interestingly, not all PT-vWD platelets are bound to $\mathrm{vWF}$, and this may partly account for the interindividual variability in platelet count and bleeding diathesis of PTvWD patients ${ }^{1}$ and mice. ${ }^{32}$

Conditions enhancing vWF levels, such as infection, surgery, pregnancy, or the treatment with DDAVP typically decrease the platelet count in type $2 \mathrm{~B} \mathrm{vWD}$ and in PT-vWD patients. ${ }^{38,39}$ Our data support the recommendation that DDAVP administration may not be advisable for PT-vWD patients ${ }^{40}$ although some patients do not experience thrombocytopenia on treatment with DDAVP ${ }^{41}$.

In conclusion, we show for the first time that constitutive binding of vWF to mutated GPIb $\alpha$ of PT-vWD megakaryocytes triggers dysregulated intracellular signaling leading to the loss of the physiological inhibition of proplatelet formation on type I collagen, and thus to ectopic platelet release in the bone marrow. This, together with the formation of a reduced number of large platelets by PT-vWD megakaryocytes and increased platelet clearance, causes thrombocytopenia. Our study clarifies the complexity of the mechanisms leading to thrombocytopenia in PT-vWD and may help to develop novel treatment options. In particular, studies to investigate whether the inhibition of GPIb $\alpha$ vWF binding may, at least partially, revert thrombocytopenia in PT-vWD are warranted.

\section{Funding}

This work was supported by a Telethon grant (GGP15063) to $P G$ and by a grant from Cariplo Foundation (2013-0717) to $A B$.

\section{Acknowledgments}

The authors thank Prof. Jerry Ware (University of Arkansas, USA) and Dr. Maha Othman (Queen's University, Canada) for the kind gift of the $\mathrm{Tg}^{W T}$ and $\mathrm{Tg}^{\mathrm{G} 233 \mathrm{~V}}$ mice, Prof. Brunangelo Falini (University of Perugia, Italy) for providing human bone marrow biopsies, Dr. Barbara Bigerna (University of Perugia, Italy) for help with immunohistochemistry of platelets in murine bone marrow, Dr. Anna Maria Mezzasoma and Dr. Emanuela Falcinelli for the measurement of VWF in plasma and cell culture medium, Dr. Francesca Milano and Dr. Giuseppe Guglielmini for help with confocal microscopy. The continued collaboration of our PTVWD patient is gratefully acknowledged. 


\section{References}

1. Othman M, Kaur H, Favaloro EJ, et al. Platelet type von Willebrand disease and registry report: communication from the SSC of the ISTH. J Thromb Haemost. 2016; 14(2):411-414.

2. Orsini S, Noris P, Bury L, et al. Bleeding risk of surgery and its prevention in patients with inherited platelet disorders. The Surgery in Platelet disorders And Therapeutic Approach (SPATA) study. Haematologica. 2017;102(7):1192-1203.

3. Suva LJ, Hartman E, Dilley JD, et al. Platelet dysfunction and a high bone mass phenotype in a murine model of platelet-type von Willebrand disease. Am J Pathol. 2008; 172(2):430-439.

4. Casari C, Du V, Wu YP, et al. Accelerated uptake of VWF/platelet complexes in macrophages contributes to VWD type 2Bassociated thrombocytopenia. Blood. 2013; 122(16):2893-2902

5. Balduini A, Pallotta I, Malara A, et al. Adhesive receptors, extracellular proteins and myosin IIA orchestrate proplatelet formation by human megakaryocytes. J Thromb Haemost. 2008;6(11):1900-1907.

6. Balduini A, Malara A, Balduini CL, Noris P. Megakaryocytes derived from patients with the classical form of Bernard-Soulier syndrome show no ability to extend proplatelets in vitro. Platelets. 2011;22(4):308311.

7. Balduini A, Malara A, Pecci A, et al. Proplatelet formation in heterozygous Bernard-Soulier syndrome type Bolzano. J Thromb Haemost. 2009;7(3):478-484.

8. Dütting S, Gaits-Iacovoni F, Stegner D, et al. A Cdc42/RhoA regulatory circuit downstream of glycoprotein $\mathrm{Ib}$ guides transendothelial platelet biogenesis. Nat Commun. 2017:8:15838.

9. Nurden P, Gobbi G, Nurden A, et al. Abnormal VWF modifies megakaryocytopoiesis: studies of platelets and megakaryocyte cultures from patients with von Willebrand disease type $2 \mathrm{~B}$. Blood. 2010;115(13):2649-2656.

10. Dunois-Lardé C, Capron C, Fichelson S, Bauer T, Cramer-Bordé E, Baruch D. Exposure of human megakaryocytes to high shear rates accelerates platelet production. Blood. 2009;114(9):1875-1883.

11. Poirault-Chassac S, Nguyen KA, Pietrzyk A, et al. Terminal platelet production is regulated by von Willebrand factor. PLoS One. 2013:8(5):e63810.

12. Nurden P, Debili N, Vainchenker W, et al. Impaired megakaryocytopoiesis in type $2 \mathrm{~B}$ von Willebrand disease with severe thrombocytopenia. Blood. 2006;108(8):25872595.

13. Malara A, Balduini A. Blood platelet production and morphology. Thromb Res. 2012;129(3):241-244.
14. Chang Y, Auradé F, Larbret F, et al. Proplatelet formation is regulated by the Rho/ROCK pathway. Blood. 2007; 109(10):4229-4236.

15. Semeniak D, Kulawig R, Stegner D, et al. Proplatelet formation is selectively inhibited by collagen type I through Syk-independent GPVI signaling. J Cell Sci. 2016; 129(18):3473-3484

16. Senis YA, Mazharian A, Mori J. Src family kinases: at the forefront of platelet activation Blood. 2014; 124(13):2013-2024.

17. Sabri S, Foudi A, Boukour S, et al. Deficiency in the Wiskott-Aldrich protein induces premature proplatelet formation and platelet production in the bone marrow compartment. Blood. 2006;108(1):134140.

18. Pecci A, Malara A, Badalucco S, et al. Megakaryocytes of patients with MYH9related thrombocytopenia present an altered proplatelet formation. Thromb Haemost. 2009;102(1):90-96.

19. Guerrero JA, Kyei M, Russell S, et al. Visualizing the von Willebrand factor/glycoprotein Ib-IX axis with a platelet-type von Willebrand disease mutation. Blood. 2009;114(27):5541-5546.

20. Giannini S, Cecchetti L, Mezzasoma AM Gresele P. Diagnosis of platelet-type von Willebrand disease by flow cytometry. Haematologica. 2010;95(6):1021-1024.

21. Bury L, Malara A, Gresele P, Balduini A. Outside-in signalling generated by a constitutively activated integrin $\alpha \operatorname{IIb} \beta 3$ impairs proplatelet formation in human megakaryocytes. PLoS One. 2012;7(4):e34449.

22. Bury L, Falcinelli E, Chiasserini D, Springer TA, Italiano JE Jr, Gresele P. Cytoskeletal perturbation leads to platelet dysfunction and thrombocytopenia in Glanzmann variants. Haematologica. 2016;101(1):46-56.

23. Giannini S, Mezzasoma AM, Leone M, Gresele P. Laboratory diagnosis and monitoring of desmopressin treatment of von Willebrand's disease by flow cytometry. Haematologica. 2007;92(12):1647-1654.

24. Bafunno V, Bury L, Tiscia GL, et al. A novel congenital dysprothrombinemia leading to defective prothrombin maturation Thromb Res. 2014;134(5):1135-1141.

25. Abbonante V, Gruppi C, Rubel D, Gross O, Moratti R, Balduini A. Discoidin domain receptor 1 protein is a novel modulator of megakaryocyte-collagen interactions. J Biol Chem. 2013:288(23):16738-16746.

26. Falcinelli E, Giannini S, Boschetti E, Gresele P. Platelets release active matrix metalloproteinase-2 in vivo in humans at a site of vascular injury: lack of inhibition by aspirin. B J Haematol. 2007;138(2):221-230.

27. Pitchford SC, Momi S, Baglioni S, et al Allergen induces the migration of platelets to lung tissue in allergic asthma. Am J Respir Crit Care Med. 2008:177(6):604-612 28. Canobbio I, Visconte C, Momi S, et al.
Platelet amyloid precursor protein is a modulator of venous thromboembolism in mice. Blood. 2017;130(4):527-536.

29. Balduini CL, Cattaneo M, Fabris F, et al Inherited thrombocytopenias: a proposed diagnostic algorithm from the Italian Gruppo di Studio delle Piastrine Haematologica. 2003;88(5):582-592.

30. Kauskot A, Poirault-Chassac S, Adam F, et al. LIM kinase/cofilin dysregulation promotes macrothrombocytopenia in severe von Willebrand disease-type 2B. JCI Insight. 2016;1(16):e88643.

31. Noris P, Biino G, Pecci A, et al. Platelet diameters in inherited thrombocytopenias: analysis of 376 patients with all known disorders. Blood. 2014;124(6):e4-e10

32. Kaur H, Corscadden K, Ware J, Othman M Thrombocytopathy leading to impaired in vivo haemostasis and thrombosis in platelet type von Willebrand disease. Thromb Haemost. 2017;117(3):543-555.

33. Falati S, Edmead CE, Poole AW Glycoprotein Ib-V-IX, a receptor for von Willebrand factor, couples physically and functionally to the Fc receptor g-chain, Fyn, and Lyn to activate human platelets. Blood. 1999;94(5):1648-1656.

34. Maxwell MJ, Yuan Y, Anderson KE, Hibbs ML, Salem HH, Jackson SP. SHIP1 and Lyn kinase negatively regulate integrin alpha IIb beta 3 signaling in platelets. J Biol Chem. 2004;279(31):32196-32204

35. Severin S, Nash CA, Mori J, et al. Distinct and overlapping functional roles of Src family kinases in mouse platelets. J Thromb Haemost. 2012;10(8):1631-1645.

36. Nakata Y, Tomkowicz B, Gewirtz AM, Ptasznik A. Integrin inhibition through Lyn-dependent cross talk from CXCR4 chemokine receptors in normal human CD34+ marrow cells. Blood. 2006; 107(11):4234-4239.

37. Pereira S, Lowell C. The Lyn tyrosine kinase negatively regulates neutrophil integrin signaling. J Immunol. 2003; 171(3):1319-1327.

38. Federici AB, Mannucci PM, Castaman G, et al. Clinical and molecular predictors of thrombocytopenia and risk of bleeding in patients with von Willebrand disease type 2B: a cohort study of 67 patients. Blood. 2009; 113(3):526-534.

39. O'Connor D, Lester W, Willoughby S, Wilde JT. Pregnancy in platelet-type VWD a case series. Thromb Haemost. 2011; 106(2):386-387

40. Gresele P, Falcinelli E, Bury L. Inherited platelet function disorders. Diagnostic approach and management. Hamostaseologie. 2016;36(4):265-278.

41. Sánchez-Luceros A, Woods AI, Bermejo E, et al. PT-VWD posing diagnostic and therapeutic challenges - small case series Platelets. 2017;28(5):484-490 